\title{
Commented translation of Erwin Schrödinger's paper 'On the dynamics of elastically coupled point systems' (Zur Dynamik elastisch gekoppelter Punktsysteme)
}

\author{
Uwe Mühlich \\ Universidad Austral de Chile, Chile
}

\section{Bilen Emek Abali}

Technische Universität Berlin, Germany

\section{Francesco dell'Isola}

Sapienza Universidad de Roma, Italy

Received 29 May 2020; accepted: 28 June 2020

\begin{abstract}
The paper 'Zur Dynamik elastisch gekoppelter Punktsysteme' by Schrödinger (1914) does not seem to have attracted the attention that it deserves. We translate it into English here and we discuss its results in detail, with a view to its possible influence in the modern theories of generalised continua. The clever solution found, in terms of Bessel functions, by Schrödinger of the problem of the vibrations of a linear infinite chain of molecules gives some important methodological hints for contemporary research.
\end{abstract}

\section{Keywords}

Generalised mechanics, metamaterials, nonlocal theories

\section{Introduction}

In his paper 'Zur Dynamik elastisch gekoppelter Punktsysteme', Erwin Schrödinger considers the vibration of an infinite mono-atomic chain with nearest-neighbour interactions. Denoting the deviation of the $n$th atom or mass point from its initial position by $\xi_{n}$, the well-known mathematical formulation of this problem reads

$$
m \frac{\mathrm{d}^{2} \xi}{\mathrm{d} t^{2}}=f\left[\xi_{n+1}-\xi_{n}\right]-f\left[\xi_{n}-\xi_{n-1}\right],
$$

with $n=0, \pm 1, \pm 2, \ldots, \pm \infty$, where $m$ indicates the mass of each atom and $f$ is the elastic stiffness of the interaction linear force between two contiguous atoms. 
As the reader might expect, this classical model of discrete physics was already well known and established at that time together with Fourier's method as the standard tool for solving this kind of problem. However, using the substitutions

$$
x_{2 n}=\sqrt{f} \frac{\mathrm{d} \xi_{\mathrm{n}}}{\mathrm{d} t}, \quad x_{2 n+1}=\sqrt{f}\left[\xi_{n}-\xi_{n-1}\right],
$$

Schrödinger ingeniously derives the general solution for arbitrary initial conditions in the form

$$
x_{n}=\sum_{k=-\infty}^{\infty} x_{k}^{0} J_{n-k}(v t)
$$

with $J_{l}$ being the Bessel function of the first kind and order $l$, initial values $x_{k}^{0}$ and $v=2 \sqrt{\frac{f}{m}}$. The result for the specific case of a single initial perturbation $\epsilon$ of the 0 th atom/mass point

$$
\xi_{n}=\epsilon J_{2 n}(v t)
$$

can be inferred easily from the general solution. It turns out that this solution shows a number of surprisingly peculiar properties. In particular, a kind of 'dissipation' effect in the chain of atoms is observed.

In fact, if one considers the natural homogenised continuum equation derived from (1), i.e. the D'Alembert wave equation, it is easily proven that for the same initial condition, the corresponding solutions for the discrete system and the D'Alembert equation differ remarkably.

The paper was published in 1914 in German in the journal Annalen der Physik. In the meantime, English has become the Lingua Franca in science and Schrödinger's contribution seems to have been neglected completely in the following literature (see e.g. [1] and in particular the section 'The Vibrating String as a Limiting Case of a Chain of Oscillators').

Here, we provide an English translation of Schrödinger's paper. One reason for which said translation is deemed necessary concerns the indisputable value of the scientific contribution that it contains.

It is undoubted that Erwin Schrödinger's contribution to physical sciences are so outstanding that the paper in which we are interested may be considered, with some good reason, to be secondary. On the other hand, the analysis that it contains seems to us still topical and may initiate an important debate among the scholars interested in continuum mechanics. In particular it seems that it can help to motivate the development of socalled generalised continuum models, in particular when evolutionary problems are considered, in which inertial parts of the action functional cannot be neglected. Indeed, we believe that some questions and information provided by Schrödinger's paper are still valuable and interesting enough to be communicated to a present-day audience.

To this aim let us recall that in 1913 a seminal paper by Ernst Hellinger (see [2-5]) clearly set up the applicability limits of the so-called first gradient continuum theory, as developed based on the fundamental contributions by Cauchy. Hellinger clearly underlined that more general models were possible and desirable, as already pointed out by Gabrio Piola, whose works Hellinger seemed to know and appreciate. This point has already been discussed extensively, and therefore we refer the interested reader to [6-9]

We do not know whether Schrödinger was fully aware of the paper by Hellinger. In fact, in $1914^{1}$ generalised continuum models had been already explored by only a few scholars. The pioneering work by Piola (1848) was described by Hellinger in his work of 1913 (see [2]) and the celebrated work by Cosserat brothers was published in 1909. Therefore, the possibility to better capture the discrete nature of a chain of molecules by such theories was not clear in the literature prior to Schrödinger's considered paper. It is not easy to understand if he, at the end, was interested at all in this specific problem. Many of the statements by Hellinger imply, on the other hand, that Hellinger himself was aware of the fact that with a higher-order theory, e.g. including non-local, strain gradient, micromorphic continua or other effects, one might be able to produce results that reflect the 'discrete' behaviour effectively shown by the results in [10].

In any case, it is certain that Schrödinger's analysis supports the conclusions that lead Hellinger to demand the development of generalised continuum theories, as envisaged by Piola. In the works by Piola and/or in the paper by Hellinger, the following are clearly stated.

(i) Every continuum model is an idealisation of an underlying discrete/discontinuous nature. Hence, 'discrete' mechanics has the right to exist individually alongside continuum mechanics. It may appear astonishing 
that at the time of Piola, and then for Hellinger and Schrödinger, discrete mechanics was considered the most 'reliable' description of nature, and that continuum mechanics was regarded by all of them as a kind of 'approximation', valid macroscopically for systems made up of a large number of particles, whereas Mach and Boltzmann had a violent controversy on this point at the beginning of the 20th century (see e.g. [11-13])

(ii) Simple continua do not include enough information about the underlying mechanical structure to account for many macroscopic phenomena, among which, for instance, we must surely include wave dispersion.

(iii) Generalised continua are designed to account for those macroscopic phenomena, which are related to an underlying microscopic structure.

The aim of the translation provided within the next section is to stay as close as possible to the original text rather than to use an elegant and elaborated English. ${ }^{2}$

\section{The translated text of Schrödinger's paper}

The translation is organized according to the individual pages and corresponding page numbers of the original paper. Footnotes are placed at the end of individual pages using the same numbering which has been used in the original paper.

\section{Page $9 / 6$}

\section{On the Dynamics of Elastically Coupled Point Systems}

Often it has been claimed ${ }^{1}$ and this is a sort of profession of faith for an atomist, that all partial differential equations of mathematical physics relating spatial and temporal variations of any physical variables (temperature, deformation, field strength, etc.) are in a rigorous sense incorrect. This is because the mathematical symbol of the differential quotient demands the limit towards arbitrarily small volumes, whereas we are convinced that we have to stop generating such 'physical' differential quotients at 'physically infinitesimal small' volumes, i.e. volumes still containing many molecules; if we were continuing the limit process further, then the corresponding quotients, which so far indeed came closer and closer to a certain limit, practically reaching it very closely, would start to vary again significantly in order to approach perhaps eventually a true limit. For the latter, despite the fact that we would not be able to measure it, these simple laws which are reflected by the partial differential equations, and which are always valid for the aforementioned 'pseudo limits', would not have validity.

Whenever we want to take the aforementioned point of view seriously, we have to fulfill two tasks: Firstly, one has to derive all differential equations, obtained in a strict sense by considering a continuous medium, now also in the sense indicated above in terms of difference equations which are deduced from a model postulating the existence of molecules.

${ }^{1}$ L. Boltzmann, Ann. d. Phys. 60. p. 231; 61. p. 790.1897.

\section{Page 917}

This task is usually not the most difficult one, in many cases it has been accomplished already ${ }^{\mathbf{1}}$. It is, however, not a rewarding task either. It only provides a proof that the atomistic vision of a phenomenon is possible, i.e. compatible with our experiences (observations) but it never proves them, the observations demand an atomistic view point. And we are at a permanent disadvantage compared with the phenomenologist who reaches the goal usually quicker by means of a simple and plausible approach, whereas we are achieving it only after a sequence of tedious and complicated thoughts which may even be perceived partly as sloppy.

Hence, atomistic theory faces a second problem, and only its solution can prove eventually that the physical atomism has the right to exist alongside with phenomenological theories. It is about finding and predicting exactly those conditions under which the differential equations, which are valid in the continuum hypothesis, yield indeed measurably incorrect results due to the atomistic nature of matter. The most important and probably only success in this direction can be found in the context of the kinetic gas theory: Temperature jump in heat conduction as well as the finite sliding in dilute gases at the channel walls are facts predictable by a molecular theory, but not by a phenomenological theory of heat transport or inner friction. 
Obviously, the only way to find such cases is to compare step by step the predictions of a continuum approach with those made by a presumably adequate molecular model for many different conditions. ${ }^{3}$ This is more difficult for the molecular models as these mechanical models possess an enormous number of degrees of freedom and the task consists of integrating the equations of motion for arbitrary initial conditions. On the other hand, as is well known, certain information about such systems can be derived without performing the integration;

${ }^{1}$ Historically, it is usually the other way around: the derivation based on atomistic ideas is in general older.

\section{Page $9 / 8$}

we owe this circumstance to the mechanistic foundation for thermodynamics, the 'Physics of Equilibrium States' by Gibbs and Ludwig Boltzmann. But, whenever it is not dealing with equilibrium states, in particular when computing spatial and temporal variations of certain coordinate functions, the general methods of statistical mechanics fail, and integration of the equations of motion seems necessary.

The in the following laid out method for integrating a well-known problem of motion, which recently gained new importance for computing specific heat capacities of solid bodies ${ }^{1}$ and effects of temperature on interference patterns of X-rays ${ }^{2}$, seems to me from this general point of view to have sufficient interest to be discussed in its own right. The problem refers to a system of mass points which in the limit converges, in the aforementioned sense, to the partial differential equation of the vibrating string, i.e. the one-dimensional wave equation. It seems to me that the solution laid out here reveals relationships between the point system's laws of motion and the propagation of waves in a one-dimensional elastic medium better than the conventional method.

The mechanical system to be studied is as follows. Finitely many point masses, all with same mass $m$, are aligned and we enumerate them by $0, \pm 1, \pm 2, \pm 3, \ldots$. Two mass points with subsequent numbers are adjacent; namely, the greater number is the right neighbour of the smaller numbered mass point. Let us count from the coordinate of a mass point being at zero along the line in one of the directions called the positive direction (which we refer to as towards the right-hand side in the sequel). Each of two neighbours shall exert a force on each other, more specifically, the force between the points $n$ and $n+1$ vanishes if they are separated by a distance $+a$;

1Born u. v. Karman, Phys. Zeitschr. 13. p. 297. 1912; see also 14. p. 15 and 65. 1913; Hans Thirring, Phys. Zeitschr. 14. p. 867. 1913; 15. p. 127 nd 180. 1914.

2P. Debye, Ann. d. Phys. (4) 43. p. 49. 1914; E. Schrödinger, Physik. Zeitschr. 15. p. 79. 1914.

\section{Page 919}

in case of a distance $a+\xi$, however, the $n+1$ th experiences a force $-f \xi$ from and, exerts a force $f \xi$ on; the $n$th point, where $f$ is constant. A stable equilibrium configuration can be defined by placing the 0 th point mass at the origin and $\pm 1 \mathrm{st}, \pm 2 \mathrm{nd}, \pm 3 \mathrm{rd} \ldots$ being at $\pm a, \pm 2 a, \pm 3 a \ldots$ We aim to analyze the motion of this now fully defined mechanical system, but starting only from initial configurations for which the distances of all mass points from their aforementioned equilibrium configuration are below a specifiable and finite bound. This ensures simultaneously that there are never any infinite forces applied to a point mass. ${ }^{1}$ We introduce as coordinates the deviation of mass points from these equilibrium positions as follows:

$$
\ldots \xi_{-n}, \quad \xi_{-n+1}, \quad \ldots, \quad \xi_{-2}, \quad \xi_{-1}, \quad \xi_{0}, \quad \xi_{1}, \ldots, \quad \xi_{n-1}, \quad \xi_{n} \ldots
$$

All values are finite at the initial time as well as after each finite time step. The equations of motion read

$$
m \frac{\mathrm{d}^{2} \xi_{n}}{\mathrm{~d} t^{2}}=f\left(\xi_{n+1}-\xi_{n}\right)-f\left(\xi_{n}-\xi_{n-1}\right), \quad n=0, \pm 1, \pm 2, \ldots
$$

Our interest in the infinite system of equations (1) is due to the fact that it converges in the limit $(a=0$; $m / a, f a$ finite) to the differential equation for the infinite and stretched string or the infinite elastic truss and, moreover, a three-dimensional generalisation of this system of equations yields in the same way the equations of the linear theory of elasticity for an infinite anisotropic medium (see the works by Born and von Karman cited previously). The principle objective of this research is to inspect especially these limit processes and the question for which cases the motion of the point system is indeed similar to the motion of the continuum. 
As is widely known, the system (1) can be integrated by using the ansatz

$$
\xi_{n}=a_{n} \exp \left(i\left(v^{\prime} t+n \phi\right)\right)
$$

${ }^{1}$ Demanding that forces in the initial configuration are bounded is not sufficient. As a counter example, consider the points $0, \pm 1, \pm 2, \pm 3, \ldots$ brought to the initial positions $0, \mp a, \mp 2 a, \mp 3 a, \ldots$, respectively. Such initial conditions are excluded here. In the case of physical applications, we are anyway solely interested in small deviations from the equilibrium configuration.

\section{Page 920}

where $\phi$ is arbitrary, $v^{\prime}$ and the $a_{n}$ are some functions of $\phi$. The most general solution of (1) can be written as a continuous sequence of solutions of type (2) (for continuously varying $\phi$ ). This method is similar to Fourier's method for the string. It has the disadvantage that in the same way as the latter, a Fourier analysis of an arbitrary initial configuration is required to assess the resulting motion, and this motion is given as a continuous sequence of normal vibrations so that the final vibration can hardly be comprehended.

The structure of (1) allows for another solution method which provides a better overview in some cases. In order to show this, we introduce instead of $\xi_{n}$ and $\dot{\xi}_{n}$ new coordinates; we denote the entities

$$
\begin{gathered}
\ldots \sqrt{f}\left(\xi_{-2}-\xi_{-1}\right), \quad \sqrt{m} \dot{\xi}_{-1}, \quad \sqrt{f}\left(\xi_{-1}-\xi_{0}\right), \quad \sqrt{m} \dot{\xi}_{0}, \\
\sqrt{f}\left(\xi_{0}-\xi_{1}\right), \quad \sqrt{m} \dot{\xi}_{1}, \quad \sqrt{f}\left(\xi_{1}-\xi_{1}\right), \quad \ldots
\end{gathered}
$$

by using the symbols

$$
\ldots x_{-3}, \quad x_{-2}, \quad x_{-1}, \quad x_{0}, \quad x_{1}, \quad x_{2}, \quad x_{3} \ldots,
$$

respectively, such that

$$
x_{2 n}=\sqrt{m} \dot{\xi}_{n}, \quad x_{2 n+1}=\sqrt{f}\left(\xi_{n}-\xi_{n+1}\right) .
$$

Quantities $x_{n}$ are actually not sufficient to describe the state of motion. They determine only the velocity and the relative positions of point masses. Since the position of the system as a whole (including a possible rigid translation which can be superimposed to any motion) is of no importance to us, we may ignore the final coordinate (we can choose an arbitrary value for some $\xi$ ). ${ }^{4}$ By rewriting (1) with the aid of the variables $x_{n}$, we obtain using the short-hand notation

$$
v=2 \sqrt{\left(\frac{f}{m}\right)},
$$

the following equation:

$$
\frac{\mathrm{d} x_{2 n}}{\mathrm{~d} t}=-\frac{v}{2}\left(x_{2 n+1}-x_{2 n-1}\right) .
$$

\section{Page 921}

Owing to (3) and (4), the identity

$$
\frac{\mathrm{d} x_{2 n+1}}{\mathrm{~d} t}=-\frac{v}{2}\left(x_{2 n+2}-x_{2 n}\right)
$$

holds for all $n$ from $-\infty$ to $+\infty$. Inspection of (5) and (6) reveals that for all $n$ from $-\infty$ to $+\infty$,

$$
\frac{\mathrm{d} x_{n}}{\mathrm{~d} t}=-\frac{v}{2}\left(x_{n+1}-x_{n-1}\right)
$$

applies.

The latter is, however, nothing else than one of two fundamental relations (by ignoring the factor $v$ ) between three Bessel functions, which follow each other with a distance of 1 regarding the order parameter. Thus, we realise that we obtain a solution by identifying one of the $x_{n}$ with an arbitrary Bessel function with argument $v t$; any other $x_{k}$ can be obtained by changing the Bessel function's parameter as much as the difference of the order parameter. 
By employing different specific Bessel functions, some interesting special cases for our problem could probably be discussed with great ease by this method. However, here we want to use it to construct the general solution for an arbitrarily given initial state. We set

$$
x_{n}=x_{k}^{0} J_{n-k}(v t), \quad n=-\infty \cdots+\infty .
$$

The $J_{n}$ are the ordinary Bessel functions of the first kind with $k$ a positive or negative integer and a constant $x_{k}^{0}$. For vanishing argument, all $J_{n}$ vanish except $J_{0}$, hence (8) is the motion starting from a very simple initial state, which arises if at $t=0$ all velocities and stresses ${ }^{5}$ vanish except one single velocity or stress, namely $x_{k}$ with the value $x_{k}^{0}$. By summing up all possible solutions, we obtain the most general solution:

$$
x_{n}=\sum_{k=-\infty}^{+\infty} x_{k}^{0} J_{n-k}(v t), \quad n=-\infty \cdots+\infty,
$$

by which the $x_{n}$ are represented in their simplest form as functions of time and their initial values $x_{n}^{0}$.

\section{Page 922}

As the most simple example for illustrating partially surprising conclusions, which can be drawn with great ease from this formula, we consider the following case. Suppose that all point masses rest initially in their equilibrium positions. Only the 0 th point mass is perturbed by $+\varepsilon$ from its equilibrium position, yet it possesses zero velocity as well. Therefore, at $t=0$, only $\xi_{0}=\varepsilon$ and all other $\xi_{n}$ as well $\dot{\xi}_{n}$ vanish. According to (3) every $x_{n}^{0}=0$ with the exception $x_{1}^{0}=\varepsilon \sqrt{f}, x_{-1}^{0}=-\varepsilon \sqrt{f}$. Hence, we obtain by using (9)

$$
x_{n}=\varepsilon \sqrt{f}\left(J_{n-1}(v t)-J_{n+1}(v t)\right),
$$

and specifically for $n=2 l$

$$
x_{2 l}=\sqrt{m} \dot{\xi}_{l}=\varepsilon \sqrt{f}\left(J_{2 l-1}(v t)-J_{2 l+1}(v t)\right)=\frac{2 \varepsilon}{v} \sqrt{f} \frac{\mathrm{d}}{\mathrm{d} t} J_{2 l}(v t),
$$

or, since at $t=0$ all $\xi$ vanish, by using (4), we obtain ${ }^{1}$

$$
\xi_{l}=\int_{0}^{t} \dot{\xi}_{l} \mathrm{~d} t=\varepsilon \int_{0}^{t} \frac{\mathrm{d}}{\mathrm{d} t} J_{2 l}(v t) \mathrm{d} t=\varepsilon J_{2 l}(v t) .
$$

Thus, in this case, all mass points as a whole generate by means of their motion a mechanical picture of all even Bessel functions of the first kind.

It is interesting to note, that a propagation with some velocity of sound cannot be observed at all for this elementary perturbation, although one would expect it, for instance, because of the analogy with the stretched string. As it is well known, a string in which a small 'hill' is generated goes back quickly to the equilibrium position at the location of perturbation. The hill is getting divided into two half-height hills traveling unattenuated in both directions at the same speed of sound.

The situation is completely different herein. As depicted in the diagrams of functions $J_{n}$ (Fig. 1), by remembering the known rules for real

\footnotetext{
${ }^{1}$ The derivation of (10) is only correct for $l \neq 0$. But it is valid as well for $l=0$, because in addition to the time integral the initial value of $\xi_{0}$, namely
} $+\varepsilon$, appears which cancels against $-\varepsilon$ from the lower integral limit.

\section{Page 923}

roots and the asymptotic expansion for large arguments,

$$
J_{n}(v t)=\sqrt{\frac{2}{\pi \nu t}} \cos \left(v t-\frac{2 n+1}{4} \pi\right),
$$




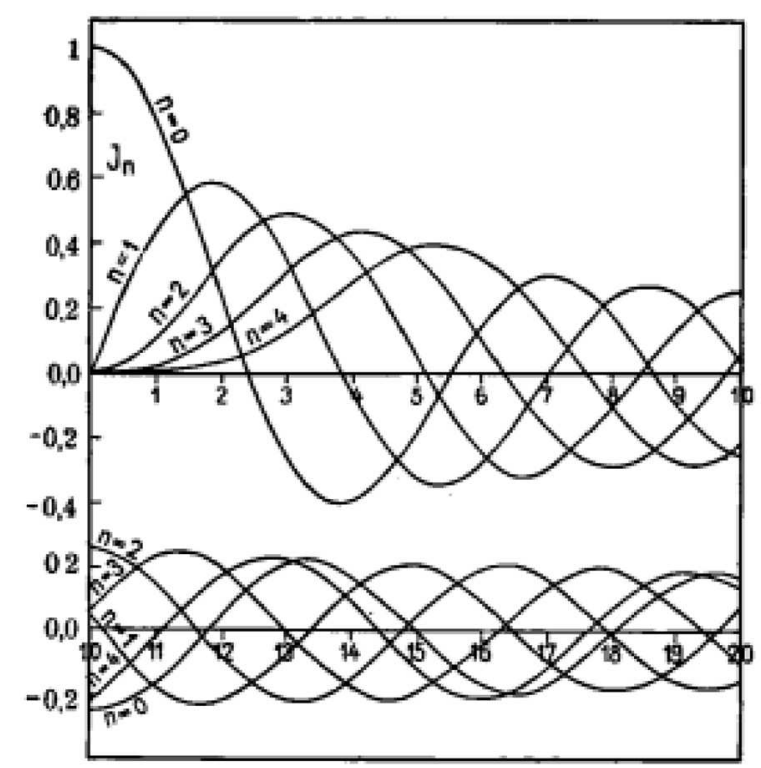

Fig. 1.

Graph der ersten fïnf Besselsehen Funktionen $J_{n}$.

Figure I. Diagram of the first five Bessel functions $J_{n}$.

we obtain the following picture [Figure 1] regarding the considered motion. The 'perturbed' mass point oscillates back passing its equilibrium position, moreover, it reverses after achieving a smaller elongation and so on. Its motion approaches a sinusoidal oscillation of a frequency being slightly greater than $v$ in the beginning; but the frequency becomes almost $v$ after a couple of oscillations, for example the time spent between the first and third passage through the equilibrium position reads

$$
\frac{3.115 \cdots+3.135 \cdots}{v},
$$

between the fourth and sixth

$$
\frac{2 \times 3.140 \ldots}{v}
$$

\section{Page 924}

The amplitude decreases gradually, after a while approximately as much as $1 / \sqrt{t}$. This phenomenon occurs because parts of the oscillation energy of a mass point is transferred gradually to its neighbours. ${ }^{1}$ After a longer period (when $v t$ is large enough to approximate Bessel functions by Eq. (11)), the vicinity of the origin oscillates almost in a kind of 'limit cycle' of our system ${ }^{2}$ : simple sinusoidal oscillations of frequency $v$, neighbouring points in anti-phase; all amplitudes, however, decrease with $1 / \sqrt{t}$ whereas this domain of nearly ordinary harmonic oscillations increases more and more.

It is noteworthy that, strictly speaking, all points start moving immediately and all move in positive direction (except the 0th). Let us consider how a somehow distant point, for instance point $l$, starts its motion. The bigger $l$ the slower its motion at the beginning and the longer it takes to reach a significant elongation. Because $J_{2 l}(v t)$ vanishes at the origin in the same way as $t^{2 l}$, its tangent order is $2 l$.

At the first maximum of $J_{2 l}$, the motion of a point reverses and passes the reference position at $v t$ being the first root of $J_{2 l}$. The latter lies (for $l>1$ ) between $\sqrt{2 l(2 l+2)}$ and $^{3} \sqrt{2(2 l+1)(2 l+2)}$, i.e. for larger values of $l, t$ is of magnitude $2 l / v$. Hence, as shown by Born and von Karman and as we will observe as well later, one can interpret the value

$$
a \sqrt{\frac{f}{m}}=\frac{a v}{2}
$$


as the speed of sound for our system, for waves that are long compared with the distance $a$ between two neighbouring points.

${ }^{1}$ This is a good example for the 'attenuating' influence of neighbours, in the same sense as observed for example in Einstein, Ann. d. Phys. 35 . p. 679. 1911. It is of importance to emphasise that this damping is occurring without any dissipative forces.

${ }^{2}$ See Born, von Karman, Physik. Zeitschr. 13. p 297. 1912.

${ }^{3}$ N. Nielsen, Handbuch der Zylinderfunktionen, p. 174. 1904.

\section{Page 925}

Such a wave starting at $t=0$ from the origin reaches the $l$ th mass point at time

$$
\frac{2 l}{v}=\frac{l a}{\frac{a v}{2}} .
$$

Hence, one can claim, that the actual oscillation starts at every point only after the corresponding sound cycle, whereas before, the motion is very small changing sign only once. Later, the period approaches more and more the value $2 \pi / \nu$.

Besides that, the maximum intensity of the motion remains always at the location of the initial perturbation, whereas the intensity decreases gradually to zero towards both directions. This whole process seems to have rather a remote resemblance with the propagation of a temperature perturbation in a linear heat conductor than with wave propagation. Given this peculiar behaviour, it seems to me important to study which relations arise anyway between our solution (9) and the general integrals solving the wave equation. Hence, we confront ourself with the question: What characteristics should the system of initial values $x_{n}^{0}$ for our system have in order to generate a motion which shows indeed a certain resemblance with the motion of a continuous elastic medium? Therefore, we follow the path of expanding $x_{n}$ in (9) in a Mac-Laurin series in terms of $v t$. To this end, we need to take the derivative of (9) once, twice, three ...times with respect to $v t$ setting always $t=0$ afterwards. In general, considering a series of type

$$
R=\sum_{l=-\infty}^{+\infty} a_{l} J_{l}(2 y)
$$

we obtain after differentiating once with respect to $y$

$$
\frac{\mathrm{d} R}{\mathrm{~d} y}=\sum_{l=-\infty}^{+\infty} a_{l}\left(J_{l-1}-J_{l+1}\right)=\sum_{l=-\infty}^{+\infty}\left(a_{l+1}-a_{l-1}\right) J_{l} .
$$

A series as in (12) is differentiated with respect to the half of the argument by replacing each coefficient by the difference between its successor and its predecessor. The resulting series is of the same form as the original one.

\section{Page 926}

Regarding the latter, we can apply to it the lemma just found to determine the sequences of coefficients for all derivatives of $R$ by constructing successively the difference quotients of the $a_{l}$ in the indicated manner

$$
\begin{array}{llll}
\ldots a_{-3}, & a_{-2}, & a_{-1}, & a_{0}, \ldots \ldots \\
\ldots \ldots & a_{-1}-a_{-3}, & a_{0}-a_{-2}, & a_{1}-a_{-1} \ldots \ldots \\
\ldots \ldots & \ldots \ldots & a_{1}+a_{-3}-2 a_{-1}, & a_{2}+a_{-2}-2 a_{0}, \ldots \\
\ldots \ldots & \ldots \ldots & \ldots \ldots & \ldots \ldots
\end{array}
$$

We find all numerical values of derivatives at $y=0$ in the " 0 th" column of this table since all $J_{n}$ vanish at $y=0$ and only $J_{0}(0)=1$. In order to apply this scheme to a series like (9), we need to replace the $a_{n}$ by the following terms:

$$
\ldots x_{n+3}^{0}, x_{n+2}^{0}, x_{n+1}^{0}, x_{n}^{0}, x_{n-1}^{0}, x_{n-2}^{0}, x_{n-3}^{0} \ldots
$$


The derivatives of $x_{n}$ regarding $v t / 2$ at $t=0$ can be found in the column $x_{n}^{0}$ of the series of differences constructed from these coefficients in the described way. Introducing the following abbreviation for the differences

$\begin{array}{lllllllll}\ldots & \Delta_{n+3}^{(1)} & \Delta_{n+2}^{(1)} & \Delta_{n+1}^{(1)} & \Delta_{n}^{(1)} & \Delta_{n-1}^{(1)} & \Delta_{n-2}^{(1)} & \Delta_{n-3}^{(1)} & \ldots \\ \ldots & \Delta_{n+3}^{(2)} & \Delta_{n+2}^{(2)} & \Delta_{n+1}^{(2)} & \Delta_{n}^{(2)} & \Delta_{n-1}^{(2)} & \Delta_{n-2}^{(2)} & \Delta_{n-3}^{(2)} & \ldots \\ \ldots & \ldots & \ldots & \ldots & \ldots & \ldots & \ldots & \ldots & \ldots\end{array}$

such that

$$
\Delta_{n}^{(1)}=x_{n-1}^{0}-x_{n+1}^{0}
$$

as well as the general recursive relation

$$
\Delta_{n}^{(k)}=\Delta_{n-1}^{(k-1)}-\Delta_{n+1}^{(k-1)}
$$

hold, we obtain

$$
\left(\frac{2}{v}\right)^{k}\left(\frac{\mathrm{d}^{k} x_{n}}{\mathrm{~d} t^{k}}\right)_{t=0}=\Delta_{n}^{(k)}
$$

The Mac-Laurin series for the $x_{n}$ reads, after using $\Delta_{n}^{(0)}$ instead of $x_{n}^{0}$ for the sake of consistency,

$$
x_{n}=\sum_{k=0}^{\infty} \frac{(v t)^{k}}{2^{k} k !} \Delta_{n}^{(k)}, \quad n=-\infty \cdots+\infty .
$$

\section{Page 927}

We have to get back to the variables $\xi_{n}$, assuming $\xi_{0}=0$ at $t=0$ for the sake of simplicity, which obviously does not imply any loss of generality. We easily find from (3)

$$
\begin{aligned}
\xi_{n} & =\xi_{0}-\frac{1}{\sqrt{f}} \sum_{l=1}^{n} x_{2 l-1} \\
& =\frac{1}{\sqrt{m}} \int_{0}^{t} x_{0} \mathrm{~d} t-\frac{1}{\sqrt{f}} \sum_{l=1}^{n} x_{2 l-1}
\end{aligned}
$$

and according to (17), taking into account (4)

$$
\xi_{n}=\frac{1}{\sqrt{f}} \sum_{k=0}^{\infty} \frac{(v t)^{k}}{2^{k} k !}\left(\Delta_{0}^{(k-1)}-\sum_{l=1}^{n} \Delta_{2 l-1}^{(k)}\right)
$$

The recursion relation (15) yields

$$
\begin{aligned}
\sum_{l=1}^{n} \Delta_{2 l-1}^{(k)} & =\sum_{l=1}^{n}\left(\Delta_{2 l-2}^{(k-1)}-\Delta_{2 l}^{(k-1)}\right) \\
& =\sum_{l=1}^{n-1} \Delta_{2 l}^{(k-1)}-\sum_{l=1}^{n} \Delta_{2 l}^{(k-1)} \\
& =\Delta_{0}^{(k-1)}-\Delta_{2 n}^{(k-1)}
\end{aligned}
$$

Thus, we obtain

$$
\xi_{n}=\frac{1}{\sqrt{f}} \sum_{k=0}^{\infty} \frac{(v t)^{k}}{2^{k} k !} \Delta_{2 n}^{(k-1)}
$$


Now we shall see how the terms $\Delta_{n}^{(k)}$, defined as difference series of the values given in (13), relate to the initial values of $\xi_{n}$ and $\dot{\xi}_{n}$ denoted by $\xi_{n}^{0}, \dot{\xi}_{n}^{0}$. According to definition (3), we can interpret the terms given in (13) themselves as a first difference series (always generated in the described manner) written below the series of the following entities:

$$
\begin{array}{ccccc}
\ldots \sqrt{f} \xi_{n+1}^{0}, & -\sqrt{m} \sum_{l}^{n+1} \dot{\xi}_{l}^{0}, & \sqrt{f} \xi_{n}^{0}, & -\sqrt{m} \sum_{l}^{n} \dot{\xi}_{l}^{0}, & \sqrt{f} \xi_{n-1}^{0} \ldots \\
\ldots x_{2 n+2}^{0}, & x_{2 n+1}^{0}, & x_{2 n}^{0}, & x_{2 n-1}^{0}, & x_{2 n-2}^{0} \ldots \\
\ldots \Delta_{2 n+2}^{(1)}, & \Delta_{2 n+1}^{(1)}, & \Delta_{2 n}^{(1)}, & \Delta_{2 n-1}^{(1)}, & \Delta_{2 n-2}^{(1)} \ldots \\
\ldots & \ldots & \ldots & \ldots & \ldots
\end{array}
$$

The lower bound of the sums $\sum_{l} \dot{\xi}_{l}^{0}$ is arbitrary; but the same choice must be made for all. Hence, we find the terms $\Delta_{2 n}^{(k)}$ in (19) we are interested in,

\section{Page 928}

in that column of the difference scheme starting with $\sqrt{f} \xi_{n}^{0}$. These columns remain unchanged, as one can see readily, if the first row is modified as follows:

$$
\ldots \sqrt{f} \xi_{n-1}^{0}, \quad \sqrt{m} \sum_{l}^{n} \dot{\xi}_{l}^{0}, \quad \sqrt{f} \xi_{n}^{0}, \quad \sqrt{m} \sum_{l}^{n+1} \dot{\xi}_{l}^{0}, \quad \sqrt{f} \xi_{n-1}^{0} \cdots
$$

The order reversal just compensates for the sign change of the summation terms. Moreover, we eliminate ${ }^{1}$ the coefficients $\sqrt{m}$ and $\sqrt{m} / a$ and consider directly the difference series of the scheme

$$
\begin{array}{ccccc}
\ldots \xi_{n-1}^{0}, & a \sum_{l}^{n} \dot{\xi}_{l}^{0}, & \xi_{n}^{0}, & a \sum_{l}^{n+1} \dot{\xi}_{l}^{0}, & \xi_{n+1}^{0} \ldots \\
\ldots \delta_{n-1}^{(1)}, & \cdot, & \delta_{n}^{(1)}, & \cdot, & \delta_{n+1}^{(1)} \ldots \\
\ldots \delta_{n-1}^{(2)}, & \cdot, & \delta_{n}^{(2)}, & \cdot, & \delta_{n+1}^{(2)} \ldots \\
\ldots & \ldots & \ldots & \ldots & \ldots
\end{array}
$$

Further explanations regarding the meaning of the $\delta_{n}^{(i)}$, introduced for those variables in the scheme which are important to us, are not really necessary. It is easy to see that

$$
\Delta_{2 n}^{(2 k-1)}=\sqrt{f} \delta_{n}^{(2 k)}, \quad \Delta_{2 n}^{(2 k)}=\frac{\sqrt{m}}{a} \delta_{n}^{(2 k+1)},
$$

Thus, we obtain from (19) after separating even and odd terms,

$$
\xi_{n}=\sum_{k=0}^{\infty} \frac{(v t)^{2 k}}{2^{2 k}(2 k) !} \delta_{n}^{(2 k)}+\frac{1}{a} \sqrt{\frac{m}{f}} \sum_{k=0}^{\infty} \frac{(v t)^{2 k+1}}{2^{2 k+1}(2 k+1) !} \delta_{n}^{(2 k+1)} .
$$

By simply rewriting and taking into account (4), the above equation takes its final form:

$$
\begin{aligned}
& \xi_{n}= \sum_{k=0}^{\infty} \frac{\left(\frac{a v t}{2}\right)^{2 k}}{(2 k) !} \frac{\delta_{n}^{(2 k)}}{a^{2 k}} \\
&+\frac{1}{\frac{a v}{2}} \sum_{k=0}^{\infty} \frac{\left(\frac{a v t}{2}\right)^{2 k+1}}{(2 k+1) !} \frac{\delta_{n}^{(2 k+1)}}{a^{2 k+1}} .
\end{aligned}
$$


This series would be identical to D'Alembert's solution of the wave equation if we were allowed to interpret the $\xi_{n}^{0}$ and $\dot{\xi}_{n}^{0}$ individually as continuous functions in terms of a coordinate $x=n a$, for example as $\xi(x)$ and $\eta(x)$ and, in addition, $a \sum_{l}^{n} \dot{\xi}_{l}^{0}$ as an indefinite integral of the form $\int^{x} \eta(x) \mathrm{d} s$, as well as the difference quotients,

$$
\frac{\delta_{n}^{(2 k)}}{a^{2 k}}, \quad \frac{\delta_{n}^{(2 k+1)}}{a^{2 k+1}},
$$

as the corresponding differential quotients of the functions $\xi(x), \int^{x} \eta(x) \mathrm{d} x$, respectively. The speed of sound, as already mentioned, becomes then $a v / 2=a \sqrt{f / m}$.

In order to convince ourselves, we remember the general solution given by D'Alembert for the wave equation

$$
\frac{\partial^{2} \xi}{\partial t^{2}}=c^{2} \frac{\partial^{2} \xi}{\partial x^{2}}
$$

is of the form

$$
\xi(x, t)=f(x-c t)+g(x+c t) .
$$

Provided that at $t=0$,

$$
\xi(x, t)_{t=0}=\xi(x), \quad\left[\frac{\partial \xi(x, t)}{\partial t}\right]_{t=0}=\eta(x),
$$

is prescribed, one can immediately gather

$$
\begin{aligned}
\xi(x, t)= & \frac{1}{2}[\xi(x+c t)+\xi(x-c t)] \\
& +\frac{1}{2 c}\left[\int^{x+c t} \eta(x) \mathrm{d} x-\int^{x-c t} \eta(x) \mathrm{d} x\right]
\end{aligned}
$$

In the corresponding Mac-Laurin series expansion with respect to $t$, all odd powers of $t$ related to the first bracket as well as all even powers of $t$ from the second bracket cancel out, and we obtain

$$
\begin{aligned}
\xi(x, t)= & \sum_{k=0}^{\infty} \frac{(c t)^{2 k}}{(2 k) !} \cdot \frac{\mathrm{d}^{2 k} \xi(x)}{\mathrm{d} x^{2 k}} \\
& +\frac{1}{c} \sum_{k=0}^{\infty} \frac{(c t)^{2 k+1}}{(2 k+1) !} \cdot \frac{\mathrm{d}^{2 k+1}}{\mathrm{~d} x^{2 k+1}} \int^{x} \eta(x) \mathrm{d} x .
\end{aligned}
$$

The structural similarity with (22) is obvious.

\section{Page 930}

From the rules for determining the coefficients $\delta_{n}^{(2 k)}$ and $\delta_{n}^{(2 k+1)}$ according to the scheme (21), it becomes obvious that the difference quotients in (22) indeed pass over to differential quotients in the limit $a=0$. More precisely, the following procedure has to be applied. Two analytic functions $\xi(x)$ and $\eta(x)$ have to be defined, which for $x=n a(n=-\infty \cdots+\infty)$ coincide with the prescribed values $\xi_{n}^{0}$ and $\dot{\xi}_{n}^{0}$, respectively. Afterwards, the limit process has to be performed such that for every intermediate value of $a$, e.g. $a^{\prime}$, at every position $n a^{\prime}$, which now coincides with the equilibrium position of a mass point, the values $\xi\left(n a^{\prime}\right)$ and $\eta\left(n a^{\prime}\right)$ are interpreted as given by $\xi_{n}^{0}$ and $\dot{\xi}_{n}^{0}$, respectively.

Of course, physically, such a limit process does not really make sense. But, the question we raised was the following: Which properties must be possessed by the system of initial values in order for the motion, represented by (9) or (22), to be similar to the motion of the stretched string for some constant a? The answer can be given with some certainty by means of the series expansions derived previously, although, as for the question, we have to forgo mathematical rigor regarding the answer. 
This will occur if the values for $\xi_{n}^{0}$ and $\dot{\xi}_{n}^{0}$ are sufficiently similar to the distribution of values of two continuous functions of $x$ and notable deviations can only be observed at distances significantly larger than $a$. In this case, at least the leading difference quotients in (22) will be rather close to the corresponding differential quotients and, as long as $t$ is not so large, that also such terms of both series for which this does not hold, gain notable influence, the motion will coincide with D'Alembert's solution based on the initial values. However, after a sufficiently long period there will always be deviations. This, by the way, follows already directly from Fourier's method mentioned earlier. With the help of the latter (see Born and von Kárman 1. c) one finds,

\section{Page 931}

that a pure sinusoidal wave of wave length $2 \pi a / \varphi^{\mathbf{1}}$ propagates with velocity

$$
a \sqrt{\frac{f}{m}} \frac{2 \sin \left(\frac{\varphi}{2}\right)}{\varphi}=\frac{a v \sin \left(\frac{\varphi}{2}\right)}{\varphi} .
$$

Hence, although small, a dispersion exists even for long waves. Consequently, an arbitrarily shaped wave will undergo a shape change after a sufficiently long time period, i.e. after its Fourier parts are separated by a finite distance due to dispersion, even if its shape is sufficiently smooth to be assembled only from long-wave Fourier parts.

However, one could argue that the conditions for varying initial conditions slowly and regularly are practically always met, because, in a molecular mechanics context, not the variables themselves, but only averages over domains containing lots of molecules possess a physical meaning. It is easy to demonstrate that all previous derivations are valid without any change if the $\xi_{n}$ are interpreted as averages of corresponding variables, in particular averages with respect to an arbitrary but once and for all fixed number of neighbouring mass points.

This reasoning is sound if averages of the $\xi_{n}$, or any temporal differential quotients of the latter, or any variables defined as linear combination of them, can be identified with observable physical quantities. Thereby, we can liberate us, for instance, from thermal motion, which appears superimposed to the longer acoustic waves in order to recognize that it has, in general, no influence on the propagation of the latter.

However, this reasoning does not apply for the propagation of a thermal perturbation itself. The simplest analogue to a thermal perturbation in our one-dimensional point system

${ }^{1} \varphi$ is the same quantity as in (2). It is the constant phase difference between neighbouring points.

\section{Page 932}

could be imagined by prescribing values for $\xi_{n}^{0}$ and $\dot{\xi}_{n}^{0}$ different from 0 over a small distance, which, however, should still be large compared with $a$, whereas all remaining points are at time $t=0$ unperturbed. The $\xi_{n}^{0}$ and $\dot{\xi}_{n}^{0}$ have to be distributed non-uniformly within this small interval, so that neighbouring mass points have opposite elongations and velocities. At $t=0$, the averages of these variables must vanish also in the perturbed part. Otherwise, there would be a displacement or a momentum of the perturbed part as a whole, which precisely should not happen. Equations (9) and (22), applied for the mean values, yield the expected result of vanishing mean values at all times. Nothing can be learned from this regarding the propagation of the perturbation because the latter depends on the squares of the $\dot{\xi}_{n}^{0}$ for which the averages do not vanish.

We do not want to dwell deeper into this question here but only comment the following: The propagation of such perturbation will not have the slightest similarity with the motion of a wave. Already the first difference quotients of the scheme (21) will not be differential quotients of analytical functions, replacing the $\xi_{n}^{0}$ and $\dot{\xi}_{n}^{0}$, because the latter will vary completely irregularly from point to point. ${ }^{6}$

All conclusions regarding the propagation of thermal perturbations, drawn from the analogy of point systems, where here the most simple representative has been considered, with continuous elastic media, as tempting as this might be ${ }^{1}$, should be considered with great care.

1See P. Debye, Ann. d. Phys. 43. p. 64 and 65. 1914; Goettinger Nachr. 1913. p. 144; Vorträge über die kinetische Theorie etc. p. 43 (Teubner 1914); M. Born, Physik. Zeitschr. 15. p. 191. 1914. Of course I assume as the same as Gentlemen Born and Debye that no finite-sized crystal can be observed as a point lattice without any imperfections; I have already used this in my manuscript about pyro- and piezoelectricity two years ago (Wien, Ber. 121 (2a), p. 1948. 1912). But opposed to Mr. Born, I do not assume that these imperfections are the essential cause of the finite heat conduction phenomenon, even if they may affect the value of the heat conductivity constant. Also I do not assume, as opposed to Mr. Debye, the necessity of a modification of Hooke's law in order to obtain a finite value for the heat conduction. 


\section{Appendix \\ The group velocity in a one dimensional elastic point array}

At first sight, the reasoning laid out here seems to be in contradiction with the results from Fourier analysis for our system of points. As already mentioned previously, it is possible to prove that a simple sinusoidal wave with wavelength:

$$
\lambda=\frac{2 \pi a}{\phi}
$$

propagates with velocity

$$
c=2 a \sqrt{\frac{f}{m}} \frac{\sin \left(\frac{\varphi}{2}\right)}{\varphi}=\frac{a v \sin \left(\frac{\varphi}{2}\right)}{\varphi},
$$

and without any shape change. For $\varphi=\pi, c$ takes its smallest value, i.e. in the case of $\lambda=2 a$. These are also the shortest waves for which it makes sense to be considered in our system. For these waves, $c$ takes the value

$$
c_{\min }=\frac{a v}{\pi}=0.658 \cdot c_{\infty}
$$

after setting the limiting value

$$
\frac{a v}{2}=c_{\infty}
$$

for long waves. It seems as if dispersion were not important, even for the shortest waves and, as if such perturbations, consisting mostly of Fourier parts with short wave lengths, must propagate with a velocity of the same magnitude as the speed of sound.

This conclusion is not correct because of the following reason. $c$ is the velocity of a homogeneous wave, traveling through our system, hence it is the so-called phase velocity. The latter, as is well known, differs in dispersive media always to some extent from the signal velocity,

\section{Page 934}

which cannot even be defined for an arbitrary signal because, it depends on the shape of the signal itself. If it consists of a 'wave head' of an otherwise homogeneous wave, the signal velocity is usually associated with the group velocity, i.e. the velocity with which the interference minima of a wave train, obtained by superimposing two simple waves with similar frequencies, travels. The group velocity is given by ${ }^{1}$

$$
v=c\left(1+\frac{\tau}{c} \frac{\mathrm{d} c}{\mathrm{~d} t}\right)^{-1}
$$

where $\tau$ is the wave period. Using instead of $c$ the wave length

$$
\lambda=c \tau
$$

one finds after a simple reformulation

$$
v=\frac{\mathrm{d}\left(\frac{1}{\tau}\right)}{\mathrm{d}\left(\frac{1}{\lambda}\right)} .
$$

In our case, we acquire with (1), (2) and (4)

$$
\frac{1}{\tau}=\frac{c}{\lambda}=\frac{\nu \sin \left(\frac{\varphi}{2}\right)}{2 \pi} ; \quad \frac{1}{\tau}=\frac{\varphi}{2 \pi a} ;
$$

hence,

$$
v=c_{\infty} \cos \left(\frac{\varphi}{2}\right)
$$


Therefore, for the shortest waves of our system $(\varphi=\pi)$, the group velocity approaches zero. This, I suppose resolves the discrepancy between the aforementioned assertions and the seemingly low dispersion resulting from Fourier analysis.

Vienna, II. Institute of Physics at the University, March 5, 1914

1The used common value:
\[ v=c\left(1-\frac{\tau}{c} \frac{\mathrm{d} c}{\mathrm{~d} t}\right) \]

(submitted on March 6, 1914)

\section{Conclusions and future perspectives}

Nowadays, there is a general awareness of the limitations of classical continua and the need for developing generalised continuum theories. Wave dispersion properties, for instance, have been investigated already in various papers using, for example, Mindlin's strain gradient theory [14, 15].

However, the problem addressed by Schrödinger has far deeper implications and there are some crucial observations, which naturally come to the mind and we want to report here.

1. In the solution given by Schrödinger, in the case of the small initial displacement of the central molecule only, all masses in the infinite chain start moving at $t=0$. This means that in the discrete infinite chain the information travels with infinite speed. However, Schrödinger observes that significant oscillations at a point at distance $l(l \gg a$, where $a$ denotes the distance between two contiguous atoms) occur only after a characteristic time $\tau=l a / c$ with $c=a \sqrt{f / m}$.

2. The very peculiar circumstances that:

(i) the maximum amplitude of oscillation propagates 'ahead' of the wave that is progressing inside the infinite array of 'atoms' leaving a wake behind it, a damped periodic motion remains at the location of the imposed initial displacement, i.e. at the mass initially displaced; and

(ii) the wake starts with an apparent irregular motion pattern and then regularizes to a damped periodic motion which is out-of-phase when two closest atoms are considered;

leads Schrödinger to find more resemblance of his solution with the propagation of a thermal perturbation than with a D'Alembertian wave propagation.

It is therefore to be expected that the 'homogenised' model for the discrete chain of molecules connected with linear elastic springs that is really capable of capturing the behaviour of the discrete system, also for initial data activating the discrete nature of considered molecule chain, should have both some hyperbolic and parabolic properties.

To be more precise: if one wants to have a continuum model whose range of applicability includes not only the possibility to consider initial data, which vary slowly at a distance of two consecutive molecules, but also initial data with 'concentrated' displacements, then the D'Alembert wave equation is to be generalised to include some features of linear parabolic equations (i.e. so-called heat equation). Possibly such an equation that can reproduce the behaviour of Schrödinger's array of atoms should belong to the class of equations similar to that proposed by Cattaneo (see [16] or [17]), but maintaining the unphysical effect of signals traveling at infinite speed.

To the best of the authors' knowledge, the contemporary contribution closest to Schrödinger's paper is due to [18]. Apparently without awareness of Schrödinger's solution but with a clear understanding of the central role of the linear mono-atomic chain as a benchmark case for continuum theories, three different initial conditions are considered by the mentioned authors, and among them exactly the initial condition discussed by Schrödinger. The solution is derived by means of a classical Laplace-Fourier approach. Charlotte and Truskinovsky intend to match the discrete case with a quasi-continuum approach. However, a number of ad hoc assumptions are required and, in addition, the authors feel the need to propose suitable approximations of their initially rather cumbersome model.

The implications of this remarkable contribution are at least twofold. On the one hand, it indicates that the problem of finding an efficient homogenised continuum description for the case addressed by Schrödinger is still far from being solved. In addition, other generalised continuum concepts exist apart from the quasi-continuum 
approach. Most of them can even be derived in different ways, e.g. by means of a bottom-up approach employing some homogenization procedure, or by pragmatically defining a consistent generalised framework according to those phenomena models derived from such a framework are supposed to capture (top-down approaches). Hence, many different ways to attack the problem exist, all of them providing their own specific potential for gaining deeper insight in the future.

\section{Notes}

1. When the translated version of Schrödinger's paper was published.

2. In this way, we follow the tradition established by William of Moerbeke in his famous translations from Greek into Latin of some of the most fundamental papers by Archimedes. One could ask why one needs a translation from a living language into another living language. The role of English language as Lingua Franca in science is not only related to the economical and political power of nations whose mother language is English, but also to some of its intrinsic features. The vocabulary used in English includes words whose roots are from the most disparate origins: Latin, Saxon, Norman, and a few others. Moreover, the British Empire imposed its own language on many dominated nations, which, on their side, influenced the standard version of English. Moulded on the structure of Latin, English soon lost declensions and complex conjugations becoming quickly an easy and flexible communication tool. Unfortunately Winston Churchill did not manage to impose a phonetic spelling of the words, so that there is no easy correspondence between the written word and the way in which it is usually pronounced. However, this is not a true problem in the process of the scientific information via written text. The destiny of the translated paper gives further evidence of the necessity of our translation. It has been often ignored and generally neglected even by those scholars who needed its content the most.

3. Here we presume that by the word 'cases' Schrödinger means situations for which the continuum description is expected to fail. For the case considered here, i.e. for the specific initial conditions he chooses for the 'molecules' chain in the lines before Equation (10), he manages to compare the solutions of d'Alembert equations with the solution he has obtained for the chain model and it turns out that d'Alembert equations, i.e. the homogenised equations for the molecules chain, cannot capture the behaviour of the discrete model for all possible initial conditions.

4. A suitable choice is, for instance, $\xi_{0}=0$.

5. The German 'Spannung' is translated as 'stress'. In this particular context, however, 'the term 'spring force', i.e. the force proportional to the elongation of the spring, seems to be more adequate.

6. We believe that the original text 'werden nichts weniger als Differentialquotienten von analytischen Funktionen sein' is a mistake or misprint.

\section{Funding}

The author(s) disclosed receipt of the following financial support for the research, authorship, and/or publication of this article: B. E. Abali's work was partly supported by a grant from the Daimler and Benz Foundation.

\section{ORCID iD}

Uwe Mühlich iD https://orcid.org/0000-0003-4097-4160

\section{References}

[1] Gallavotti, G. The Elements of Mechanics (Theoretical and Mathematical Physics). Berlin: Springer, 2013.

[2] Hellinger, E. Die allgemeinen Ansätze der Mechanik der Kontinua. Encyklopädie der mathematischen Wissenschaften 1913; 5.

[3] Eugster, SR, and dell'Isola, F. Exegesis of the Introduction and Sect.I from "Fundamentals of the Mechanics of Continua" by E. Hellinger. Z Angew Math Mech 2017; 97(4): 477-506.

[4] Eugster, SR, and dell'Isola, F. Exegesis of Sect. II and III.A from "Fundamentals of the Mechanics of Continua" by E. Hellinger. Z Angew Math Mech 2018; 98(1): 31-68.

[5] Eugster SR, and dell'Isola F. Exegesis of Sect. III.b from "Fundamentals of the mechanics of continua" by E. Hellinger. $Z$ Angew Math Mech 2018; 98(1): 69-105.

[6] dell'Isola, F, Andreaus, U, and Placidi, L. At the origins and in the vanguard of peridynamics, non-local and higher-gradient continuum mechanics: An underestimated and still topical contribution of Gabrio Piola. Math Mech Solids 2015; 20(8): 887-928.

[7] dell'Isola, F, Della Corte, A, and Giorgio, I. Higher-gradient continua: The legacy of Piola, Mindlin, Sedov and Toupin and some future research perspectives. Math Mech Solids 2017; 22(4): 852-872.

[8] dell'Isola, F, Andreaus, U, Cazzani, A, et al. The complete works of Gabrio Piola: Volume II commented english translation. 1st ed. New York: Springer, 2018.

[9] dell'Isola, F, Maier, G, Perego, U, et al. The Complete Works of Gabrio Piola: Volume I Commented English Translation - English and Italian Edition. New York: Springer, 2014. 
[10] Schrödinger, E. Zur Dynamik elastisch gekoppelter Punktsysteme. Ann Phys 1914; 349(14): 916-934.

[11] Floyd, J, and Shieh, S (eds.) Future Pasts: The Analytic Tradition in Twentieth-century Philosophy. Oxford: Oxford University Press, 2001.

[12] Kartsev, V. The Mach-Boltzmann Controversy and Maxwell's Views on Physical Reality. Dordrecht: Springer Netherlands, 1981, pp. 199-205.

[13] Stöltzner, M. Vienna indeterminism: Mach, Boltzmann, Exner. Synthese 1999; 119(1/2): 85-111.

[14] Papargyri-Beskou, S, Polyzos, D, and Beskos, D. Wave dispersion in gradient elastic solids and structures: A unified treatment. Int $J$ Solids Struct 2009; 46(21): 3751 - 3759.

[15] Rosi, G, Placidi, L, and Auffray, N. On the validity range of strain-gradient elasticity: A mixed static-dynamic identification procedure. Eur J Mech A/Solids 2018; 69: 179-191.

[16] Cattaneo, C. Sur une forme de l'équation de la chaleur éliminant le paradoxe d'une propagation instantanée. Comptes Rendus 1958; 247(4): 431-433.

[17] Müller, I, and Ruggeri, T. Rational Extended Thermodynamics (Springer Tracts in Natural Philosophy). New York: Springer, 1998.

[18] Charlotte, M, and Truskinovsky, L. Lattice dynamics from a continuum viewpoint. J Mech Phys Solids 2012; 60(8): 1508-1544. 